\title{
Treatment of Hepatitis C with Direct-Acting Antivirals does not Induce Significant Arrhythmias
}

André Gustavo da Silva Rezende, ${ }^{1}{ }^{\circledR}$ Edmundo Pessoa Lopes, ${ }^{2}$ Andrea Doria Batista, ${ }^{2}$ Norma Arteiro Filgueira, ${ }^{2}$ Williane Emanuelle Rodrigues Costa, ${ }^{3}$ Poline Maria de Sousa Felix, ${ }^{3}$ Brivaldo Markman Filho

Cardiology Section, Hospital das Clínicas, Universidade Federal de Pernambuco (UFPE), ${ }^{1}$ PE - Brazil

Gastroenterology Section, Hospital das Clínicas (UFPE), ${ }^{2}$ PE - Brazil

Medical Students - Center of Medical Sciences (UFPE), ${ }^{3}$ PE - Brazil

\section{Abstract}

Background: Chronic Hepatitis C (CHC) therapy with direct-acting antivirals (DAAs) has high efficacy and safety, but some cases of bradyarrhythmias have been described.

Objective: To evaluate heart rhythm disorders during DAA treatments.

Methods: Forty-eight patients with CHC (mean 61 years of age; 56\% males; 73\% HCV genotype 1) were evaluated before and during treatment with DAAs, analyzed by a resting 12-lead ECG [PR, QRS, and QT corrected (QTc) intervals measured] and a 24-h-Holter system, to evaluate the heart rate (HR) and the occurrence of arrhythmias. The Student's t-test or the Wilcoxon-Mann-Whitney test for continuous, independent variables were performed with a statistically significant $\mathrm{p}$-value $<0.05$.

Results: The electrocardiographic parameters before and during treatment were: PR interval (147.2 \pm 15.6 vs 144.9 $\pm 15.6 \mathrm{~ms} ; \mathrm{p}=0.21)$, QTc interval ( $427 \pm 22.3$ vs $421.7 \pm 25.3 \mathrm{~ms} ; \mathrm{p}=0.24)$, minimum HR ( $52.7 \pm 8.4$ vs $53.2 \pm 8.5$ bpm; $\mathrm{p}=0.49)$, median HR (74.2 \pm 10.4 vs $75.2 \pm 9 \mathrm{bpm} ; \mathrm{p}=0.83)$, and maximum HR $(117.4 \pm 16.8$ vs $117.9 \pm 16.3 \mathrm{bpm} ; \mathrm{p}=$ 0.25). These parameters proved to be similar among 11 beta-blockers or 22 ribavirin users. During treatment, the 21 cirrhotic patients presented significantly lower median HRs (72.1 \pm 9.0 vs $77.9 \pm 8.2 \mathrm{bpm}$; $\mathrm{p}=0.02)$ and maximum HRs $(108.9 \pm 15.2$ vs. $125.1 \pm 13.2$ bpm, p < 0.0001) through a 24-h-Holter monitoring than the patients without cirrhosis. No clinically relevant arrhythmias were detected.

Conclusion: DAAs do not significantly influence heart rate or induce significant cardiac arrhythmias in patients with CHC.

Keywords: Sofosbuvir/ therapeutic use; Hepatitis C/treatment; Arrhythmias Cardiacs; Antiviral Agents; Drug Resistance Viral.

\section{Introduction}

Hepatitis C Virus (HCV) infection affects approximately 71 million people, accounting for 400,000 deaths per year worldwide. ${ }^{1}$ More than $70 \%$ of these patients develop Chronic Hepatitis $\mathrm{C}(\mathrm{CHC})$, progressing with different degrees of liver impairment. ${ }^{2}$ $\mathrm{CHC}$ has been reported as a cause of atherosclerotic heart disease and cardiomyopathies. ${ }^{3,4}$ Advanced liver fibrosis may also contribute to the development of structural, hemodynamic, and electrophysiological cardiac changes, which characterize cirrhotic heart disease. ${ }^{5}$ Furthermore, antiviral treatment of $\mathrm{HCV}$ with interferon or antivirals may be associated with cardiac arrhythmias. ${ }^{6}$

The introduction of direct acting antivirals (DAAs), including sofosbuvir, allowed for patients to be treated with CHC exclusively via the oral route, without 
interferon (IFN-free). These medications have an excellent efficacy and safety profile. ${ }^{7}$ However, after the beginning of the use of DAA regimens in $2012,{ }^{8}$ the FDA reported nine cases of severe bradyarrhythmia occurring on the first days of therapy. ${ }^{9}$ Another study reported the occurrence of three similar cases in a French reference center, among more than 400 patients treated with DAAs. ${ }^{10}$ The nine patients from the FDA report who developed bradyarrhythmia were undergoing amiodarone therapy. In the French report, only one patient was treated with this drug, one patient used a beta-blocker, and the third patient did not use antiarrhythmic drugs. These facts have raised questions about the possible effect of sofosbuvir on patient heart rates (HR).

The objective of this study was to evaluate the HR changes and other clinical and electrocardiographic parameters in patients with $\mathrm{CHC}$ during DAA treatment, including sofosbuvir, analyzed by a 12-lead electrocardiography (ECG) and a 24-h ambulatory electrocardiographic monitoring (Holter system),

\section{Methods}

\section{Subjects}

The present study was developed at the Hospital das Clínicas, Universidade Federal de Pernambuco (HCUFPE), Brazil. We included patients older than 18 years of age with $\mathrm{CHC}$, characterized by serum anti-HCV positivity for more than 6 months and confirmed by the presence of serum HCV-RNA, who met the criteria for treatment with the new DAAs established under the Clinical Protocol and Therapeutic Guidelines for Hepatitis $C$ and Coinfections set forth by the Brazilian Ministry of Health (PCDT-2015). ${ }^{11}$ This protocol states that patients should present at least one of the following criteria: hepatic fibrosis (documented METAVIR $\geq$ F2), clinical signs of liver cirrhosis, HIV coinfection, extrahepatic hepatitis $C$, hematological malignancies, idiopathic thrombocytopenic purpura, or previous transplant of solid organs.

Patients taking amiodarone were excluded, as were patients with severe heart disease, persistent cardiac arrhythmias [atrial tachycardia, atrial fibrillation or flutter, and second- or third-degree atrioventricular (AV) block], cardiac pacemakers, and chronic renal failure with a glomerular filtration rate $\leq 30 \mathrm{~mL} / \mathrm{min}$.

\section{Methodology}

The patients were interviewed and examined at the Hepatology outpatient clinic, in the Gastroenterology Section at HC-UFPE. After study inclusion, $10 \mathrm{~mL}$ of venous blood was also collected through the vacutainer system to measure the hematologic, virologic, and liver function tests.

The laboratory tests were performed by automated method at the Central Laboratory of HC-UFPE. HCV RNA and HCV genotype were determined by real-time polymerase chain reaction using COBAS AmpliPrep/ COBAS TaqMan (version 2, Roche, Pleasanton, CA, USA) with a detection limit of $15 \mathrm{IU} / \mathrm{ml}$. Hepatic fibrosis was assessed through the histopathology of a liver biopsy specimen, using the METAVIR classification, point shear wave elastography (pSWE), or Fib4 and APRI serologic scores. The percutaneous liver biopsies were performed using a $16 \mathrm{G} \times 90 \mathrm{~mm}$ Menghini needle in, at most, two punctures. The pSWE was determined by Acoustic Radiation Force Impulse (ARFI) using ACUSON S2000 ${ }^{\mathrm{TM}}$ ultrasound (Siemens, Muenchen, Germany). The APRI and FiB-4 scores were calculated as described by Wai et al. ${ }^{12}$ and Sterling et al. ${ }^{13}$ Advanced liver fibrosis was considered when METAVIR F3 or F4, FIB-4 $>3.5$, and APRI $>1.5$.

Cirrhosis was defined as the presence of clinical and laboratory signs of hepatic impairment, as well as alterations in the hepatic architecture and/or signs of portal hypertension observed in ultrasonography or upper gastrointestinal endoscopy.

The cardiovascular evaluation involved the investigation of specific symptoms, including dyspnea, chest pain, palpitation, and syncope, as well as risk factors, such as systemic arterial hypertension (SAH), diabetes mellitus (DM), previous heart disease, coronary artery disease (CAD), cardiac arrhythmias, and the use of cardiovascular drugs, particularly antiarrhythmic agents.

The 12-lead ECG was recorded using an EP 12 eletropágina ${ }^{\circledR}$ digital apparatus (Dixtal Biomédica ${ }^{\circledR}$, São Paulo, Brazil). The ECG traces were evaluated by a single examiner with experience in the method. The measurements of the intervals (in milliseconds) were obtained manually, with extended traces, if necessary, at minimum intervals of approximately $10 \mathrm{~ms}$. For the heart rate (HR) measurement, the RR interval was determined in at least three consecutive QRS complexes and the mean value was used to calculate the final value. The same approach was used to calculate the QT interval. In the 
sinus rhythm, normal HR was defined as 60-100 bpm. HR $<60$ bpm was considered sinus bradycardia and HR $>100$ bpm was considered sinus tachycardia. The normal PR interval was considered to be 120-200 ms. AV block was classified as first degree (PR interval $>200 \mathrm{~ms}$ ), second degree type I (progressive increase of PR interval before a blocked P wave), type II (P wave blocked without previous PR interval increase), 2:1 (two $P$ waves for each QRS complex), advanced (two or more blocked P waves), and third degree (complete AV block). Intraventricular conduction delays were classified as bundle branch block (right and left) and left fascicular block (anterior and posterior). The QT intervals were corrected using the Bazett formula, defined by the measured QT interval divided by the square root of the RR interval in seconds (QT/ $\sqrt{R R})$. Corrected QT (QTc) intervals between 350 and $450 \mathrm{~ms}$ in males and 350 and $460 \mathrm{~ms}$ in females were considered normal. ${ }^{14}$ QTc intervals above $480 \mathrm{~ms}$ were considered a significant increase.

The Cardios ${ }^{\circledast}$ system (São Paulo, Brazil) was used for 24-h Holter monitoring. Three simultaneous leads were digitally recorded using Cardiolight ${ }^{\circledR}$ recorders, and the traces were analyzed using the Cardiosmart ${ }^{\circledR}$ software. The analysis was performed by a single examiner experienced in the method. The following parameters were evaluated: minimum, median, and maximum HR; presence of frequent supraventricular arrhythmias (number of supraventricular ectopic beats $>30 / \mathrm{h}$ ), supraventricular tachycardia lasting for $>10 \mathrm{~s}$, recurrent ventricular arrhythmias (number of ectopic beats $>30 / h$ ), non-sustained or sustained ventricular tachycardia, the presence of pauses for $>3 \mathrm{~s}$, and impairment in atrioventricular or intraventricular conduction.

The patients included in the study underwent the first cardiac evaluation with ECG and 24-h Holter monitoring. Subsequently, the patients started treatment schemes with DAAs (sofosbuvir combined with either daclatasvir or simeprevir \pm ribavirin) for $12-24$ weeks. Reassessment during therapy was scheduled $10-40$ days after the first dose of the antivirals and included an additional medical consultation, ECG, and 24-h Holter monitoring. Patients were informed about the onset of warning symptoms, including palpitation, shortness of breath, chest pain, dizziness, and syncope. In these cases, they should seek immediate medical care to detect clinical changes outside the evaluation periods. All patients underwent cardiovascular monitoring until 30 days after the end of therapy.

\section{Statistical analysis}

The statistical analysis was performed using the package SPSS Statistics ${ }^{\circledR}$, version 21 (IBM®). The descriptive analysis included clinical and laboratory variables. Continuous data were presented as mean \pm standard deviation (SD) or median and interquartile range (IQR) in the case of the presence or not of normal distribution, respectively. The ECG parameters (HR, PR interval, QRS duration, and QTc interval) and Holter monitoring parameters (minimum, median, and maximum HR) were compared before and during $\mathrm{HCV}$ treatment. Inferential analysis of quantitative variables was conducted using homogeneity and normal distribution tests (Kolmogorov-Smirnov and ShapiroWilk tests) to determine the use of parametric tests (Student's t-test) or non-parametric tests (Wilcoxon and Mann-Whitney tests). The chi-square test and Fisher's exact test were used for the categorical variables. P-values $<0.05$ were considered statistically significant.

\section{Ethical approvals and consent to participate}

The study was approved by the Ethics and Research Committee on Human Beings, UFPE Health Sciences Center (approval no: 1.566.240), and the patients who agreed to participate in the study were asked to sign an informed consent form.

\section{Results}

From December 2015 to August 2017, 53 patients were evaluated. Two patients were excluded from the study because of the presence of chronic arrhythmias (atrial fibrillation and flutter, respectively) and three patients were excluded because they failed to attend the subsequent evaluations. Of the three patients who did not undergo reevaluation, two finished the therapy without complications. The third patient discontinued antiviral therapy without medical advice; however, no complications were found to justify the discontinuation of the treatment. Therefore, 48 patients comprised the final study sample and underwent cardiovascular monitoring.

The following drug combinations were used: sofosbuvir, daclatasvir, plus ribavirin - 21 patients (43.8\%); sofosbuvir plus simeprevir - 15 patients (31.2\%); sofosbuvir plus daclatasvir - 11 patients $(22.9 \%)$; and sofosbuvir, simeprevir plus ribavirin - 1 patient (2.1\%).

The mean age of the patients was $61 \pm 9.8$ years (range $37-81)$, and most patients $(56.3 \%)$ were males. 
Two patients (4.3\%) had a positive serology for HIV. The evaluation of liver fibrosis stages was obtained by liver biopsy in 34 patients, by hepatic elastography in one patient, and by APRI and FIB 4 scores in 13 patients. Thirty-three patients $(68.8 \%)$ presented advanced liver fibrosis, while 21 patients $(43.8 \%)$ had a clinical diagnosis of liver cirrhosis. The HCV genotype 1 was present in $73 \%$ of the sample. Eleven patients (22.9\%) were using betablockers. Clinical and laboratory data on liver disease are summarized in Table 1.

Specific symptoms were present in $25 \%$ of the patients in the initial cardiovascular evaluation. Dyspnea was the most common symptom, alone or combined with other complaints, and was reported by nine patients $(18.8 \%)$, although none of these cases included cardiac or respiratory changes. With respect to the history of cardiovascular problems, SAH and DM were observed in $56.3 \%$ and $39.6 \%$ of the sample, respectively. Only one patient had a diagnosis of CAD with previous myocardial revascularization surgery.

Four patients $(8.3 \%)$ reported symptoms in the reassessment performed after an average of $18.7 \pm 9.4$ days of treatment initiation. Three $(6.2 \%)$ of these patients reported palpitations, and one reported dyspnea, which had no association with diagnosed cardiac changes. The systolic blood pressure $(131.6 \pm 20.8$ vs. $128.2 \pm 15.8$, p $=0.3)$ and diastolic blood pressure $(76.8 \pm 10.3$ vs. 78 $\pm 10.5, \mathrm{p}=0.4$ ) before and during treatment were not significantly different.

The baseline ECG data showed changes in 14 patients $(29.2 \%)$. Sinus bradycardia was the most common finding, found in six patients $(12.5 \%)$, with $\mathrm{HR}$ values ranging from 50 to $60 \mathrm{bpm}$. First-degree AV block was observed in two patients $(4.2 \%)$, intraventricular conduction delays in six (12.5\%) patients, and increased QTc interval in four patients; however, none of these patients had QTc values > $480 \mathrm{~ms}$. The second ECG recording performed during therapy was considered abnormal in 14 patients (29.2\%), particularly in four patients $(8.3 \%)$ with a long QTc interval, two of whom did not present this alteration at baseline. However, the increase in the QT interval was considered mild in three of the four patients (from 461 to $471 \mathrm{~ms}$ ) and significant in only one patient (464 ms in the first examination and 494 $\mathrm{ms}$ in the second examination). Furthermore, this increase was not correlated with significant changes in the T-wave morphology or the presence of polymorphic ventricular arrhythmias analyzed by 24-h Holter monitoring, and treatment interruption was not necessary. This patient finished therapy without suffering cardiac complications.
The 24-h Holter monitoring did not indicate a significant difference in the minimum, medium, and maximum $H R$ values and the presence of recurrent supraventricular or ventricular arrhythmias when comparing the periods before and during treatment (Table 2). Two patients without ventricular arrhythmias at baseline had a high frequency of ventricular extrasystoles during therapy, corresponding to $13.3 \%$ and $3.4 \%$ of the total heart beats, respectively. The increase in the number of ventricular ectopic beats in these patients was not associated with the presence of symptoms or other cardiovascular alterations, and interruption of treatment was not indicated. No cases of supra-ventricular or ventricular tachycardia or significant bradycardia with pauses exceeding $3 \mathrm{~s}$ were observed. None of the patients using DAAs had cardiovascular complications that required the discontinuation of treatment.

Subgroup analysis was performed to assess the effect of beta-blockers or advanced liver disease on ECG and 24-h Holter monitoring parameters. Eleven patients were on beta-blockers, with propranolol being used by six of them. The use of beta-blockers did not affect the ECG and HR parameters evaluated by $24-\mathrm{h}$ Holter monitoring before and after therapy (Table 3). Moreover, the use of ribavirin by 22 patients did not affect the ECG and HR parameters evaluated by 24 -h Holter monitoring before and after therapy. No differences were found before and during treatment: minimum HR $(52.48 \pm 7.34$ vs $54.76 \pm$ $9.15 \mathrm{bpm}, \mathrm{p}=0.06)$, median HR $(73.67 \pm 10.48$ vs $74.48 \pm$ $10.13 \mathrm{bpm}, \mathrm{p}=0.58)$, and maximum HR (114.71 \pm 14.24 vs $112.33 \pm 16.45 \mathrm{bpm}, \mathrm{p}=0.36)$, respectively. Similarly, there were no significant differences in these parameters when comparing the periods before and during antiviral therapy in the 21 patients with cirrhosis.

However, during the treatment with DAAs, the 21 patients with cirrhosis presented significantly lower median HR and maximum HR values analyzed by 24-h Holter monitoring than did the patients without cirrhosis (Table 4). This difference was possibly due to a slight decrease in maximum HR during treatment in cirrhotic patients and the slight increase in median HR and maximum HR in non-cirrhotic patients (Table 4).

\section{Discussion}

Our findings did not demonstrate the presence of significant changes in the ECG parameters and HR evaluated by dynamic ECG (24-h Holter monitoring) during treatment with DAAs. To date, no studies 
have been found in the literature that evaluate 24-h Holter monitoring during treatment with DAAs for CHC patients.

At the beginning of the $\mathrm{CHC}$ treatment with these new antivirals, some reported cases of severe bradyarrhythmia that were associated with prior use of amiodarone, and the clinical complication was explained by the interaction between this drug and DAAs, particularly sofosbuvir. ${ }^{15}$ Therefore, some studies have investigated the cardiovascular safety profile of DAAs. In effect, Caldeira et al. performed a meta-analysis that included six large studies and 1,625 patients with $\mathrm{CHC}$ on antiviral therapy and found no significant differences in the rate of occurrence of cardiac arrhythmias between patients receiving sofosbuvir and those receiving other treatment regimens without this drug. The presence of arrhythmias was defined by the ECG records or compatible symptoms, including palpitations, pre-syncope, or syncope, and was reported in only three of these studies, with an overall risk of approximately $2 \%$ in patients using sofosbuvir. ${ }^{16}$

In effect, Durante-Mangoni et al. found that 26 patients on sofosbuvir regimens did not present changes in HR on serial ECG, including patients using beta-blockers, which suggested that this drug does not increase the risk of developing bradyarrhythmia during treatment with DAAs. These authors reported that the QTc interval increased in the first week after beginning therapy and returned to baseline levels during treatment. ${ }^{17}$ Nonetheless, changes in HR were not found in the 11 patients from our study who were using beta-blockers, analyzed by 24-h Holter monitoring.

Additionally, there were four cases of increased QTc interval during treatment among our patients, although the interruption of antiviral therapy was not necessary, since this increase was either mild or moderate (QTc below $480 \mathrm{~ms}$ ) in three patients and significant in only one case, which was not associated with ventricular arrhythmias or changes in T-wave morphology analyzed by 24-h Holter monitoring. Hagiwara et al., ${ }^{18}$ found cardio-vascular changes related to antiviral therapy in three cases $(3.3 \%)$ among 91 patients using sofosbuvir plus ledipasvir. According to these authors, one of the patients presented bradycardia and increased QT interval, another patient developed atrial fibrillation, and the third patient presented a prolonged QT interval associated with previous heart failure. The three patients presented clinical improvement after discontinuing the treatment. ${ }^{18}$ In contrast, Biomy et al. ${ }^{19}$ evaluated patients using sofosbuvir plus simeprevir and found no cardiovascular events or changes in clinical, electrocardiographic, and echocardiographic parameters within 6 months after treatment. ${ }^{19}$

In the present study, two of the non-cirrhotic patients who did not present ventricular arrhythmia before therapy did show a high rate of monomorphic ventricular arrhythmia when analyzed by 24-h Holter monitoring during treatment. The occurrence of ventricular arrhythmia was also reported by Nirei et al. in two cirrhotic patients with spontaneous resolution. ${ }^{20}$ The asymptomatic monomorphic ventricular tachycardia occurred at the beginning of therapy with sofosbuvir plus ledipasvir, leading to the interruption of treatment. These authors did not investigate the mechanisms involved in the onset of the arrhythmias but recommended caution in the use of these drugs in patients with advanced liver disease. ${ }^{20}$

In the present study, HR was evaluated in patients with cirrhosis, and in this subgroup, the use of DAAs did not induce the development of significant bradyarrhythmia. The median and maximum HR values evaluated by $24-\mathrm{h}$ Holter monitoring were lower in patients with liver cirrhosis than in non-cirrhotic patients, particularly during treatment, with a decrease in HR values compared with baseline values. Moreover, the use of DAAs appears to increase the median and maximum HR in non-cirrhotic patients. It is noteworthy that these findings were not associated with the use of beta-blockers, which is prescribed for portal hypertension in cirrhotic patients. Likewise, no differences were found in HR during ribavirin use, although this drug may induce hemolytic anemia, which can increase the HR. ${ }^{21}$

The degree of hepatic dysfunction may affect cardiac and autonomic function, causing chronotropic incompetence and an increase in the QT interval. ${ }^{22}$ Other authors have reported a lower HR in response to physical or pharmacological stress in cirrhotic patients, as evidenced by maximum HR values in provocation tests, such as physical effort in cycle ergometry ${ }^{23}$ or the use of dobutamine. ${ }^{24}$ Two mechanisms that lead to this alteration are decreased sensitivity of cardiac beta-adrenergic receptors as a consequence of the chronic hyperadrenergic state observed in cirrhotic cardiomyopathy (down-regulation) and the impairment of other factors involved in sympathetic signal transduction, including the receptor and the activity of $\mathrm{G}$ protein and adenyl cyclase, resulting in a decrease in cAMP levels. ${ }^{25}$ These changes in HR have not yet been described when using 24-h Holter monitoring 
in patients with CHC during treatment with DAAs. The mechanism by which DAAs sensitize chronotropic incompetence in patients with cirrhosis or even increase the HR in non-cirrhotic individuals remains unclear.

The results of this study reinforce the cardiovascular safety profile of the treatment of $\mathrm{CHC}$ with DAAs. The widespread use of this therapy in several countries and the low number of complications in the medical literature strongly suggest that the reported complications are due to other factors, including the concomitant use of antiarrhythmics, as well as existing cardiac problems or other cardiovascular risk factors. The strength of this study was the use of 24-h Holter monitoring to evaluate the dynamic characteristics of $\mathrm{HR}$, which allowed for the detection of cardiac arrhythmias, even in the absence of symptoms. In addition, strict parameters were used to define ECG changes, including abnormal QTc intervals $>450 \mathrm{~ms}$ instead of values starting from $440 \mathrm{~ms}$, which is the cut-off used in most studies.

One limitation of this study was the low number of patients in the sample due to the high cost of treatment with DAAs, which restricted the access to therapy offered by the Brazilian public health system to patients with more advanced liver disease or comorbidities. This public policy has been reviewed in recent years when treatment became available to all CHC patients.

Another limitation of this study may have been the completion of the evaluation by ECG and 24-h Holter parameters between 10 and 14 days after the start of antiviral therapy, which may have caused some changes in HR that have not been diagnosed. For that reason, long-term continuous ECG monitoring, immediately after beginning treatment, could be more effective in detecting cardiac arrhythmias.

\section{Conclusion}

Our results demonstrate that $\mathrm{HCV}$ treatment with DAAs, including sofosbuvir, was not associated with significant clinical and ECG changes, and did not affect the main ECG or 24-h Holter parameters, even in patients using beta-blockers and ribavirin. Long QT interval and ventricular arrhythmia occurred in isolation and did not require the discontinuation of therapy. However, the use of DAAs appears to decrease the median and maximum HR in patients with cirrhosis analyzed by 24-h Holter monitoring. Further studies with larger sample sizes are necessary to confirm our findings.

\section{Author contributions}

Conception and design of the research: Lopes E, Markman-Filho B, Filgueira N, Rezende A. Acquisition of data: Rezende A, Costa W, Felix P. Analysis and interpretation of the data: Rezende A, Lopes E, Batista A, Markman-Filho B. Statistical analysis: Rezende A, Lopes E. Writing of the manuscript: Rezende A. Critical revision of the manuscript for intellectual content: Lopes E, Batista A, Markman-Filho B.

\section{Potential Conflict of Interest}

No potential conflict of interest relevant to this article was reported.

\section{Sources of Funding}

There were no external funding sources for this study.

\section{Study Association}

This article is part of the thesis of master submitted by André Gustavo da Silva Rezende, from Universidade Federal de Pernambuco.

\section{Ethics approval and consent to participate}

This study was approved by the Ethics Committee of the CCS/UFPE under the protocol number 1.566.240. All the procedures in this study were in accordance with the 1975 Helsinki Declaration, updated in 2013. Informed consent was obtained from all participants included in the study. 


\section{References}

1. World Health Organization. Hepatitis C: fact sheet. (Accessed 11 January 2020). Available from http://www.who.int/mediacentre/factsheets/fs164/en/.

2. Spearman CW, Dusheiko GM, Hellard M, Sonderup M. Hepatitis C. Lancet. 2019; 394(10207):1451-66.

3. Ambrosino P, Lupoli R, Di Minno A, Tarantino L, Spadarella G, Tarantino $\mathrm{P}$, et al. The risk of coronary artery disease and cerebrovascular disease in patients with hepatitis C: A systematic review and meta-analysis. Int J Cardiol. 2016 221:746-54.

4. Prati D, Poli F, Farma E, Picone A, Porta E, Mattei C, et al. Multicenter study on hepatitis $C$ virus infection in patients with dilated cardiomyopathy. J Med Virol. 1999; 58(2):116-20.

5. Zardi EM, Abbate A, Zardi DM, Dobrina A, Margiotta D, Tassell BW, et al. Cirrhotic Cardiomyopathy. JAC. 2010; 56(7):539-49.

6. Sonnenblick M, Rosin A. Cardiotoxicity of interferon. A review of 44 cases. Chest. 1991; 99(3):557-61.

7. Pecoraro V, Banzi R, Cariani E, Chester J, Villa E, D'Amico R, et al. New Direct-Acting Antivirals for the treatment of patients with Hepatitis C virus infection: A Systematic Review of Randomized Controlled Trials. J Clin Exp Hepatol. 2019; 9(4):522-38.

8. Stedman CA. Current prospects for interferon-free treatment of hepatitis C in 2012. J Gastroenterol Hepatol. 2013; 28(1):38-45.

9. FDA Hepatitis Update - Important safety information: Harvoni and Sovaldi (Accessed 19/12/2015). Avaiable from http://content.govdelivery. com/accounts/USFDA/bulletins/f97c71.

10. Fontaine H, Duboc D, Pol S. Bradyarrhythmias associated with sofosbuvir treatment. N Engl J Med. 2015; 373(2):1886-8.

11. Brasil.Ministério da Saúde. Clinical Protocol and Therapeutic Guidelines for Hepatitis C and Coinfections 2015. (Accessed 01 December 2016). Available from http://www.portalsaude.saude.gov.br.

12. Wai CT, Greenson JK, Fontana RJ, Kalbfleisch JD, Marrero JA, Conjeevaram HS, et al. A simple noninvasive index can predict both significant fibrosis and cirrhosis in patients with chronic hepatitis C. Hepatology. 2003;38(2):518-26.

13. Sterling RK, Lissen E, Clumeck N, Sola R, Correa MC, Montaner J, et al. Development of a simple noinvasive index to predict significant fibrosis in patients with HIV/HCV coinfection. Hepatology. 2006; 43(6):1317-25.
14. Postema PG, Wilde AA. The measurement of the QT interval. Curr Cardiol Rev. 2014; 10(3):287-94.

15. Back DJ, Burger DM. Interaction between amiodarone and sofosbuvirbased treatment for hepatitis C virus infection: Potential Mechanisms and Lessons to be Learned. Gastroenterology. 2015; 149(6):1315-7.

16. Caldeira D, Rodrigues FB, Duarte MM. Cardiac harms of sofosbuvir : systematic review and meta-analysis. Drug saf. 2017; 41(1):77-86.

17. Durante-Mangoni E, Parrella A, Vitrone M, Rago A, Pafundi PC, Nigro $\mathrm{G}$, et al. Electrophysiological adverse effects of direct acting antivirals in patients with chronic hepatitis C. J Clin Pharmacol. 2017; 57(7):924-30.

18. Hagiwara S, Nishida N, Watanabe T. Outcome of combination therapy with sofosbuvir and ledipasvir for chronic type C liver disease. Oncology. 2017; 92(suppl 1):3-9.

19. Biomy R, Abdelshafy M, Abdelmonem A, Elenin HA, Ghaly G. Effect of chronic hepatitis $C$ virus treatment by combination therapy on cardiovascular system. Clin Med Insights Cardiol. 2017; 11:1-9.

20. Nirei K, Nakamura H, Matsuoka S, Yamana Y, Yoda S, Hirayama A, et al Ventricular tachycardia as a complication of ledipasvir and sofosbuvir treatment for HCV infection. Intern Med. 2017; 56(7):787-90.

21. Bodenheimer HC, Lindsay KL, Davis GL, Lewis JH, Thung SN, Seeff LB, et al. Tolerance and efficacyof oral ribavirin treatment of chronic hepatitis C: a multicenter trial. Hepatology. 1997; 26(2):473-7.

22. Mozos I. Arrhythmia risk in liver cirrhosis. World J Hepatol 2015;7(4):662-672.

23. Wong F, Girgrah N, Graba J, Allidina Y, Liu P, Blendis L. The cardiac response to exercise in cirrhosis. Gut. 2001;49(2):268-75.

24. Rudzinski W, Waller AH, Prasad A, Sood S, Gerula C, Samanta A, et al. New index for assessing the chronotropic response in patients with end-stage liver disease who are undergoing dobutamine stress echocardiography. Liver Transpl. 2012; 18(3):355-60.

25. Zenghua Ma, Miyamoto A, Lee SS. Role of altered beta-adrenoceptor signal transduction in the pathogenesis of cirrhotic cardiomyopathy in rats. Gastroenterology. 1996; 110(4):1191-8 